\title{
Confinement-induced vitrification in polyethylene terephthalate
}

\author{
F. J. Baltá Calleja, ${ }^{2}$ G. Di Marco, ${ }^{1}$ A. Flores, ${ }^{2}$ and M. Pieruccini ${ }^{1}$ \\ ${ }^{1}$ Istituto per i Processi Chimico-Fisici Sezione di Messina, CNR, Via La Farina 237, I-98123 Messina, Italy \\ ${ }^{2}$ Instituto de Estructura de la Materia, CSIC, Serrano 119, 28006 Madrid, Spain
}

(Received 1 March 2007; published 4 June 2007)

\begin{abstract}
Dynamic mechanical thermal analysis performed on cold-drawn polyethylene terephthalate (PET), cold crystallized (annealed) in the temperature interval $100-140{ }^{\circ} \mathrm{C}$, reveals the presence of marginally glassy domains above the annealing temperature $T_{a}$. This suggests that the thermodynamic force driving crystallization causes the structural arrest of some noncrystalline domains. The latter thus need a temperature higher than $T_{a}$ to completely defreeze. Differential scanning calorimetry supports this point of view. Analogous investigations on unoriented PET, cold crystallized in the same conditions, do not show the same peculiarities; thus, chain orientation is relevant to vitrification. This phenomenology is first cast in the language of thermodynamics by introducing an excess chemical potential $\delta \mu$ describing the presence of structural constraints in the amorphous domains and the effect of chain orientation. For a first test of this picture, the orientation contribution to $\delta \mu$ is calculated by means of the Gaussian chain model (this implicitly assumes that $\delta \mu$ is related to the density fluctuations). The resulting expression is then used to discuss the structural differences between cold-drawn and unoriented PET samples reported in the literature.
\end{abstract}

DOI: 10.1103/PhysRevB.75.224201

\section{INTRODUCTION}

In addition to its multiple industrial applications, polyethylene terephthalate (PET) is of great interest as a model system for basic research. ${ }^{1}$ In particular, nanostructured crystalline-amorphous phase separation in PET has been extensively studied under a rather wide range of different crystallization conditions. ${ }^{2}$ The relatively high glass transition temperature and the slow crystallization rates typical of this material are central to this point. Furthermore, the possibility of obtaining an oriented glass from unoriented glassy PET by just drawing at room temperature (cold drawing) opens up the possibility of studying properties where the mean chain orientation plays a significant role (e.g., phase transitions).

Dynamic mechanical thermal analysis (DMTA) and differential scanning calorimetry (DSC) studies show that crystal growth during annealing of cold-drawn PET may cause the vitrification of the amorphous regions by progressive confinement. ${ }^{3}$ On the contrary, a similar phenomenology, wherever present, does not show up during or after the annealing of unoriented PET. Thus, a question regarding the assessment of the role of a mean chain orientation in the vitrification of the amorphous regions during the crystallization process arises.

The dependence on the mean chain orientation is also found for the mechanical performance of the glass. As a particularly interesting example, the data analysis of hardness and elastic modulus by means of microindentation ${ }^{4,5}$ suggests the occurrence of a reinforced amorphous phase in annealed cold-drawn PET in contrast to the unoriented material.

The present paper offers a description of the above observations in terms of the well established concepts of statistical thermodynamics. Some of our previously published data are used to test the validity of this model, together with a number of additional experimental results. We will emphasize the role of the constraints on long-wavelength density fluctuations occurring within the interlamellar regions, as a relevant aspect of the mechanism underlying the vitrification process.
PACS number(s): 61.82.Pv, 61.82.Rx, 64.70.Pf, 05.20.-y

It will be shown that the amorphous regions are driven toward a glassy behavior when the characteristic linear dimensions of the confining volume approach the density autocorrelation length of the "melt" in the absence of confinement. The difference between isotropic and cold-drawn semicrystalline samples, with regard to the vitrification process, can be associated with the vectorial character that chain orientation confers to the density autocorrelation length.

Some information concerning materials and experimental techniques is briefly reported in Sec. II. The key experimental results, illustrated and discussed to some extent in Sec. III, serve as a basis for the development of a general scheme to describe the observations (Sec. IV). In doing this, we will resort to the statistical mechanical description of liquids close to the glass transition. ${ }^{6}$ The structural constraints in these liquids are expressed through an excess chemical potential affecting their mobility. The actual calculations for the present case will be performed using the Gaussian chain model, ${ }^{7}$ which allows us to introduce chain orientation in a rather straightforward way. The calculation details are reported in the Appendix. Finally, in Sec. V, we will use the results of the theory to discuss and analyze the structural data of annealed cold-drawn PET available in literature.

\section{EXPERIMENT}

Oriented glassy PET $\left(M_{w} \sim 20.000 \mathrm{~g} / \mathrm{mol}\right)$ was prepared from unoriented glassy strips of $\sim 0.5 \mathrm{~mm}$ thickness, from Goodfellow (ES 301465). The length of the strips increases naturally by a factor $\kappa \simeq 4$ due to cold drawing. ${ }^{3-5}$ On the other hand, the sample shrinks perpendicularly to the drawing direction by a factor of $\sim 0.3$, which can be considered negligible as compared to $\kappa$. The samples were then isothermally crystallized (annealed) from the glassy state at selected temperatures $T_{a}$ under nitrogen flux. The cold-drawn samples were clamped during crystallization. The annealing times were 9 and $2 \mathrm{~h}$ for the unoriented and the cold-drawn materials, respectively. 


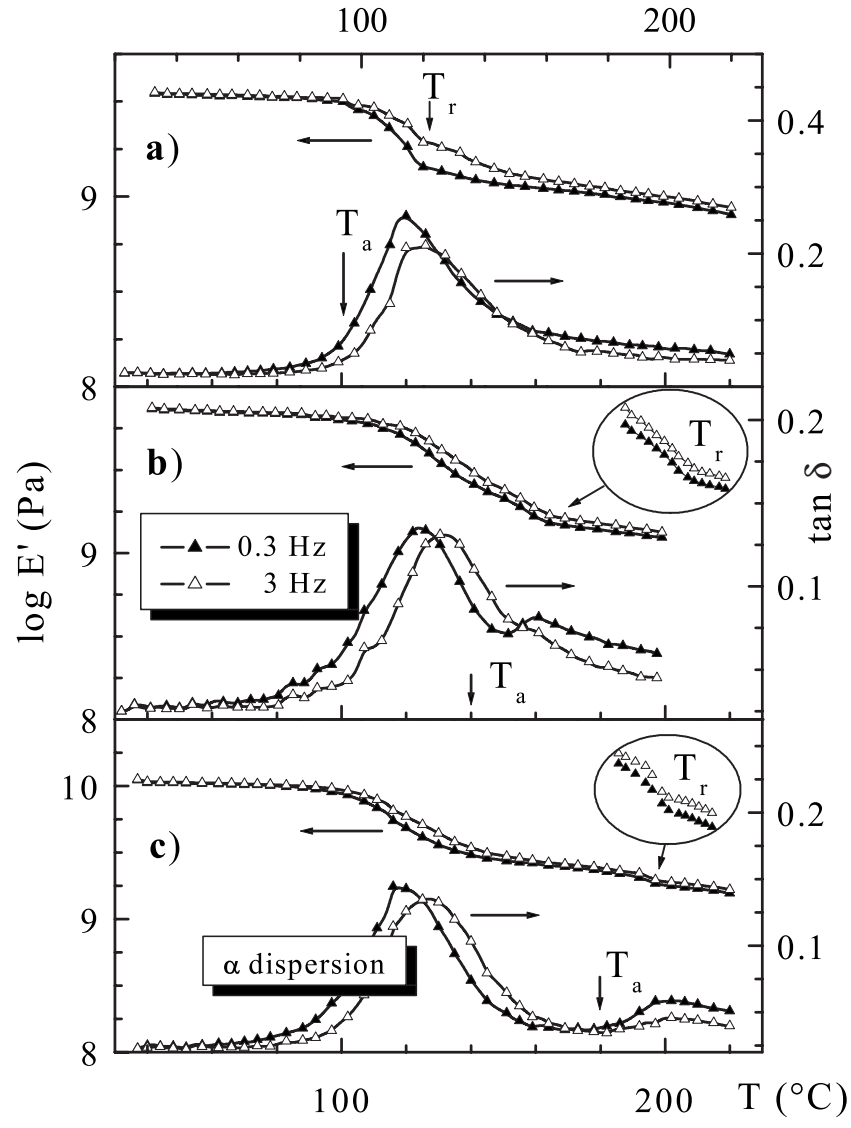

FIG. 1. DMTA scans at 0.3 and $3 \mathrm{~Hz}$ obtained with a heating rate of $2{ }^{\circ} \mathrm{C} / \mathrm{min}$ on cold-drawn PET annealed at the temperatures $T_{a}$ of (a) $100{ }^{\circ} \mathrm{C}$, (b) $140{ }^{\circ} \mathrm{C}$, and (c) $180{ }^{\circ} \mathrm{C}$ (see Ref. 3). The left axes report the logarithm of the storage moduli, and the right axes the corresponding loss tangents. The $\alpha$ dispersion region is well evidenced in the $T_{a}=180{ }^{\circ} \mathrm{C}$ spectrum.

The mechanical properties of both the starting isotropic and the cold-drawn samples were already investigated by means of a dynamical mechanical thermal analyzer of Polymer Laboratories (MKII). ${ }^{3}$ Results for the annealed colddrawn material $\left(T_{a}=100,140\right.$, and $\left.180{ }^{\circ} \mathrm{C}\right)$ are also taken from Ref. 3. In all cases, the DMTA measurements were carried out in bending geometry, single cantilever configura- tion. The heating rate was set to $2{ }^{\circ} \mathrm{C} / \mathrm{min}$ with an accuracy of $0.1{ }^{\circ} \mathrm{C}$; the probed frequencies were 0.3 and $3 \mathrm{~Hz}$.

Calorimetric measurements have been carried out with a Perkin-Elmer Pyris 1 differential scanning calorimeter calibrated with indium and zinc standards. The thermograms of annealed cold-drawn PET have been reported previously. ${ }^{3}$ In the present study, data concerning unoriented PET annealed at $120^{\circ} \mathrm{C}$ are presented. In all cases, the DSC scans were performed using a heating rate of $40{ }^{\circ} \mathrm{C} / \mathrm{min}$ under constant nitrogen flux.

\section{RESULTS}

Figure 1 reports the DMTA patterns of the cold-drawn samples annealed at 100,140 , and $180^{\circ} \mathrm{C}$ (see Ref. 3). One broad dispersion is observed in the temperature interval $\sim 100-160{ }^{\circ} \mathrm{C}$, consisting of an $\alpha$ relaxation which, in the low- $T_{a}$ annealed samples, is convoluted with a recrystallization process starting at about $T_{r}=T_{a}+20^{\circ} \mathrm{C}$. The latter, unlike the $\alpha$ process, does not depend on the DMTA probing frequency. Details can be found in Ref. 3; here, it is important to note that the temperature range spanned by the $\alpha$ process includes the annealing temperatures $T_{a}=100$ and $140^{\circ} \mathrm{C}$. The onset of the $\alpha$ dispersion region does not appear to depend significantly on the annealing temperature and, thus, on the crystallinity (see Table I). This means that the glassy domains seem to contain regions of poor mechanical properties whose features are independent of $T_{a}$. In addition, it is worth noticing that the onset of the $\alpha$ process shifts to lower temperatures in the case of the unoriented material (see Fig. 2; data from the oriented $T_{a}=100{ }^{\circ} \mathrm{C}$ and $T_{a}$ $=140{ }^{\circ} \mathrm{C}$ samples are included for comparison); this shift is even larger for the cold-drawn unannealed sample $\left(T_{g, o r} \simeq 50{ }^{\circ} \mathrm{C}\right.$, i.e., $\sim 20{ }^{\circ} \mathrm{C}$ lower than in the unoriented glass; this can probably be connected to the presence of internal stresses induced as a consequence of cold drawing). Previous dielectric measurements in isotropic semicrystalline PET suggest that the broad $\alpha$ process observed embraces two relaxation processes: the low-temperature one is associated with the interstack or interspherulitic amorphous material and the high-temperature process is ascribed to amorphous domains undergoing relaxation in a regime of confinement. ${ }^{8}$

TABLE I. Long period $L$, lamellar thickness $l_{c}$, and volume crystallinity $\alpha_{V}$ as functions of the annealing temperature $T_{a}$ of initially cold-drawn PET. $\phi$ is the angle between the mean drawing direction and the normal to the basal planes. The lamellar thickness $l_{c}$ is calculated from $\alpha_{V}$ and $L$ (data from Ref. 4).

\begin{tabular}{|c|c|c|c|c|c|}
\hline $\begin{array}{l}T_{a} \\
\left({ }^{\circ} \mathrm{C}\right)\end{array}$ & $\begin{array}{c}L \\
(\mathrm{~nm})\end{array}$ & $\begin{array}{c}\phi \\
(\operatorname{deg})\end{array}$ & $\alpha_{V}$ & $\begin{array}{c}l_{c} \\
(\mathrm{~nm})\end{array}$ & $\begin{array}{c}l_{a} \\
(\mathrm{~nm})\end{array}$ \\
\hline 100 & 11 & 55 & 0.22 & 1.4 & 5 \\
\hline 120 & 11.1 & 50 & 0.28 & 2 & 5 \\
\hline 140 & 11.3 & 46 & 0.35 & 2.8 & 5.2 \\
\hline 160 & 11.7 & 44 & 0.41 & 3.5 & 5 \\
\hline 180 & 12 & 41 & 0.48 & 4.4 & 4.8 \\
\hline 200 & 12.4 & 40 & 0.55 & 5.2 & 4.2 \\
\hline 220 & 14 & 38 & 0.63 & 7 & 4.1 \\
\hline 240 & 15.8 & 37 & 0.72 & 9.1 & 3.5 \\
\hline
\end{tabular}






FIG. 2. DMTA scans at $0.3 \mathrm{~Hz}$ obtained with a heating rate of $2{ }^{\circ} \mathrm{C} / \mathrm{min}$ on cold-drawn and unoriented PET in the $\alpha$ dispersion regions. Data from Ref. 3, except for the unoriented $T_{a}=120^{\circ} \mathrm{C}$ sample.

Recent dielectric studies in cold-crystallized oriented PET suggest that the nature of its $\alpha$ relaxation is similar to that of the high-temperature process in the isotropic material. ${ }^{9}$ The occurrence of fast relaxing amorphous regions, away from the conditions of highly confined dynamics, seems to be inhibited in the case of the oriented material.

The processes occurring at $T_{r} \simeq T_{a}+20{ }^{\circ} \mathrm{C}$ are associated with lamellar destabilization. More explicitly, they are related to the onset of simultaneous melting and recrystallization processes, involved in the rearrangement of a structure tending to reach thermodynamic stability in an environment where the temperature is steadily increasing, as in a DMTA run.

The heat absorption associated with the increased mobility characterizing marginally unstable semicrystalline structures can also be detected by DSC, as shown in Fig. 3 (for a thorough account of this reorganization process, see Refs. 10 and 11). The exothermal process at the high- $T$ side of the peak at $T_{r}$ is, in general, faster in the cold-drawn samples than in the unoriented ones. This is shown in the inset in Fig. 3 for $T_{a}=120{ }^{\circ} \mathrm{C}$ (the baseline of the isotropic sample thermogram has been suitably modified to make the comparison easier); note the "ordinary" glass transition at $T_{g} \simeq 85^{\circ} \mathrm{C}$ in the isotropic sample, which is absent in the cold-drawn annealed one. We finally note that the drop in the base line accompanying the recrystallization onset at $T_{r}$ seems to be more pronounced in the cold-drawn samples annealed at $T_{a}$ $<160{ }^{\circ} \mathrm{C}$.

\section{THEORY}

\section{A. Phenomenological approach}

It appears that the most representative result is derived from the DMTA. It is rather peculiar, in fact, that, e.g., the annealing temperature $T_{a}=100{ }^{\circ} \mathrm{C}$ is located at the onset of the $\alpha$ process. This means that there is a temperature range above $T_{a}$ where some (marginally) glassy domains are still



FIG. 3. DSC scans at a heating rate of $40{ }^{\circ} \mathrm{C} / \mathrm{min}$ on colddrawn PET annealed at (a) $100{ }^{\circ} \mathrm{C}$, (b) $120^{\circ} \mathrm{C}$ (c) $140{ }^{\circ} \mathrm{C}$, (d) $160{ }^{\circ} \mathrm{C}$, and (e) $180{ }^{\circ} \mathrm{C}$. The arrows indicate the enhanced exothermal contribution to the heat flux immediately following the heat absorption associated with the destabilization of the lamellae. The inset shows a comparison between cold-drawn and isotropic samples annealed at $T_{a}=120{ }^{\circ} \mathrm{C}$ in the region where recrystallization sets in. Data from Ref. 3 except for the unoriented $T_{a}$ $=120^{\circ} \mathrm{C}$ sample.

frozen. Yet, the possibility of providing the small-angle x-ray scattering (SAXS) data of Table I relies on the presence of a crystal-amorphous stacking periodicity. This periodicity cannot develop within a glassy system; moreover, as shown in Fig. 2, prior to any annealing procedure, the onset of the glass transition in cold-drawn PET is found approximately at $T=40{ }^{\circ} \mathrm{C}$. Hence, we are led to conclude that the transition of the amorphous phase toward a glassy state may only take place after the crystal phase starts to develop. At low enough crystallization temperatures, the generalized thermodynamic force responsible for crystal growth is, thus, intense enough to induce constraints to chain mobility in the amorphous domains (via pinning and chain connectivity), which need a temperature higher than $T_{a}$ to disappear.

On the other hand, it is worth pointing out that this vitrification process, as mentioned above, is not evident in the case of unoriented samples: The annealing temperatures are always at the upper bound of the $\alpha$ dispersion or higher (see Fig. 2 for $T_{a}=120^{\circ} \mathrm{C}$ ). This is an important fact, because it points to the relevance of chain orientation in the vitrification process.

In order to build up a convenient picture for the description of the vitrification, we start by introducing the concept of "defect" as any chain conformational feature (e.g., torsions, entanglements, etc.) preventing a monomer from accommodating in an elementary crystalline cell. Crystal growth implies that these defects are, in general, pushed away from the crystallizing regions, concentrating gradually in the amorphous domains as crystallization proceeds. Due to pinning and chain connectivity, in fact, the number of defects is a quasiconserved quantity. When a defect moves away from a crystallization front to allow a chain unit to be part of a crystal, in general, it diffuses away in the same amorphous domain due to chain connectivity. In this position, the defect 
will actually oppose the propagation of a different crystalline front with a probability that increases when the extension of the amorphous domain decreases. Eventually, the volume of the amorphous regions becomes so small that structural arrest occurs.

This picture implies that some correlation length should be relevant in the vitrification process. Since the concentration of defects is coupled to density and the motion of defects (i.e., structural rearrangement) is, in general, associated with density fluctuations, we expect that the density autocorrelation length could be a key quantity for understanding our experimental observations, as indeed will be evident below with Eq. (6). However, the most relevant aspect of the model is that the diffusion of defects at long distances, away from crystalline fronts, must be assisted by density fluctuations of comparable wavelength. When the volume of an amorphous region is progressively reduced by the crystals, the longwavelength density modes cannot be active and the defects remain trapped in the amorphous islands, preventing further crystallization. The model describes, in fact, a confinementinduced vitrification mechanism, either as a consequence of confinement of defects or, which is assumed to be the same, as a consequence of the establishment of upper limits to the wavelength of the "active" density fluctuation modes.

With respect to confinement, as a means to probe the relevant length scales at the glass transition, the present situation differs significantly from others considered in the literature (an exception is given by Ref. 12). Here, (i) the confining material is the same as the vitrifying one, and (ii) confinement effects develop progressively as a characteristic confinement length decreases. The last point means that a sort of crystal-quasiglass coexistence condition sets in.

Regarding the DSC analysis, the peculiar character of the exothermal contributions illustrated in Fig. 3 for $T_{a}$ $\leqslant 140^{\circ} \mathrm{C}$ can be interpreted in light of our picture as follows. When the temperature rises enough above $T_{a}$, the crystalline domains destabilize and the configurational constraints weaken. The conditions keeping the interlamellar regions into a glassy state break down, however, at a temperature $T_{r}$ about $20^{\circ} \mathrm{C}$ higher than that where the glass was previously formed. As can be seen from the storage moduli in the DMTA patterns in Fig. 1, further crystallization then takes place from a condition of nonequilibrium (i.e., a just defrozen glass). In contrast, for the $T_{a} \geqslant 160{ }^{\circ} \mathrm{C}$ annealed samples, crystallization at $T_{r} \simeq T_{a}+20{ }^{\circ} \mathrm{C}$ occurs in the presence of amorphous domains which are not in the (marginally) glass state because $T_{r}$ falls above the $\alpha$ dispersion region. This would explain why the exothermal contributions are more pronounced in the low- $T_{a}$ samples.

The vitrification process taking place within the amorphous regions for low $T_{a}$ is reminiscent of a picture where the generalized force driving lamellar growth (an affinity, in the sense of irreversible thermodynamics) in order to reach a minimum of the overall thermodynamic potential is contrasted by an increase of this potential due to the glass formation. From basic principles of thermodynamics, the establishment of conformational constraints implies, indeed, an increase of the thermodynamic potential of the amorphous component. Upon heating, when these conformational constraints relax, then the conditions for keeping the amorphous



FIG. 4. Liquid and solid branches of the chemical potential $\mu$ as functions of the temperature (solid lines). The dashed line relates to a "constrained liquid" state describing the effect of the vitrification; it is tangent to the liquid line as it meets it. The dotted line is an extrapolation; it crosses the solid line at the apparent melting point $T_{a p p}^{\infty}$

regions in a glassy state immediately drop, and the generalized force is then free to drive further crystallization toward a more stable thermodynamic condition for the whole system. When this happens faster, the larger is the magnitude of the force that has been balanced by vitrification.

Resorting to the ideas introduced by Adam and Gibbs for the description of systems close to the glass transition, ${ }^{6}$ we assign an apparent chemical potential $\mu_{a p p}$ to the amorphous phase in the presence of conformational constraints, related to the ordinary chemical potential $\mu_{a}$ by

$$
\mu_{\text {app }}=\mu_{a}+\delta \mu>\mu_{a},
$$

where $\delta \mu$ describes the effect of the constraints (and/or chain orientation). Note that $\delta \mu$ must be a function of the mean chain orientation in the amorphous domains. Let us remember that confinement-induced vitrification is observable only in the cold-drawn annealed samples. A (rough) estimate of $\delta \mu$ in the special case of the interlamellar amorphous regions will be done in what follows by means of a Gaussian chain model (see Doi and Edwards, ${ }^{7}$ and more specifically the work of Vilgis et al. ${ }^{13}$ ).

Figure 4 shows the confinement vitrification process represented in the phase diagram. The "glass" branch is not, of course, a true thermodynamic line. In fact, it depends on the kinetics of glass formation, so it has been drawn in the figure (see dashed line) just as a reference for the concepts developed above. The inset in Fig. 4 illustrates the statistical mechanical meaning of $\delta \mu$, as will be shown below.

\section{B. Statistical mechanical approach: Effect of mean chain orientation}

The scope of the present section is to cast the ideas introduced previously in the framework of statistical mechanics. In this respect, the connection with thermodynamics is achieved through the partition function $Z$, which is relevant 
in the description of a system in contact with a heat bath. Depending on the extensive variables which the heat bath allows to fluctuate, both the associated thermodynamic potential $F$ and the partition function $Z$ take different forms, though always related by the expression

$$
F=-k_{B} T \ln Z,
$$

with $k_{B}$ the Boltzmann constant and $T$ the absolute temperature.

With reference to known strategies for handling the problem of the structural glass transition, ${ }^{14} \mathrm{Z}$ is assumed to be factorizable as

$$
Z \simeq Z_{v i b} Z_{c o n f}
$$

where $Z_{v i b}$ is associated with the vibrational degrees of freedom of the system, whereas $Z_{\text {conf }}$ is connected to the spectrum of allowed conformations. This separation (independence) of fluctuation processes occurring on such different time and length scales, and the corresponding split $F \simeq F_{v i b}$ $+F_{\text {conf }}$, has the nature of an approximation. In this framework, the structural glass transition of a liquid or a polymer melt is associated with changes in $Z_{\text {conf }}$. Thus, the chemical potential difference $\delta \mu$ defined through Eq. (1) has to be related to the conformational part $F_{\text {conf }}$ of the free energy.

In order to estimate the effects of a mean chain orientation and of the presence of mesoscopic structural constraints on $Z_{c o n f}$, we will resort to the Gaussian chain model. ${ }^{7}, 13 \mathrm{Al}-$ though rather crude, this model will turn out to be capable of accounting for the relevant mechanism governing the confinement-induced vitrification.

Within this framework, a central quantity is the segment distribution function $\Psi_{t}$ describing the probability that the chains filling a volume $V$ take a given conformation. An outline of its derivation is given in the Appendix; here we report its approximate expression in the representation of the (complex) amplitudes $c_{\mathbf{k}}$ of the density normal modes with wave vector $\mathbf{k}$ :

$$
\Psi_{t}\left\{c_{\mathbf{k}}\right\} \propto \exp \left[-\frac{V a^{2}}{24 c} \sum_{\mathbf{k}}\left(\mathbf{k}_{\perp}^{2}+\kappa k_{\|}^{2}+\xi^{-2}\right) c_{\mathbf{k}} c_{-\mathbf{k}}\right],
$$

where $c$ is the average segment number density, $a$ is the average bond length, and $\kappa$ is the orientation parameter $(\kappa$ $>1$ if the chains are preferentially oriented along the direction labeled with \|, whereas $\kappa=1$ for an isotropic melt); $\xi$ is a function of the excluded volume interaction parameter $v$ (see the Appendix),

$$
\xi=1 / \sqrt{8 c a v}
$$

and turns out to be the density autocorrelation length in an isotropic melt. Indeed, the density profile $\left\langle\delta c^{2}\right\rangle \equiv\langle c(\mathbf{r}) c(\mathbf{0})\rangle$ $-c^{2}$, around a $\delta$-like perturbation of the density at the origin, can be directly calculated through the expression for $\Psi_{t}$, Eq. (4), to yield [cf. Eqs. (A12)-(A14) in the Appendix]

$$
\left\langle\delta c^{2}\right\rangle=\frac{3 c}{\pi a^{2}\left[\mathbf{r}_{\perp}^{2}+\kappa^{-1} r_{\|}^{2}\right]^{1 / 2}} \exp \left[-\xi^{-1}\left(\mathbf{r}_{\perp}^{2}+\kappa^{-1} r_{\|}^{2}\right)^{1 / 2}\right]
$$

Note that if the chain is elongated $(\kappa>1)$, the density autocorrelation length along the mean chain direction increases to the value $\xi \sqrt{\kappa}$. This suggests that the existence of a mean chain orientation can be relevant to the vitrification process.

Turning back to Eq. (4), representing $\Psi_{t}$ in the $c_{\mathbf{k}}$ space results in an easier way of introducing confinement constraints to the relaxation dynamics of the density fluctuations. The partition function $Z_{c o n f}$ is the integral of $\Psi_{t}\left\{c_{\mathbf{k}}\right\}$ with respect to the mode amplitudes $c_{\mathbf{k}}$. Due to the approximations adopted, this reduces to the calculation of ordinary Gaussian integrals:

$$
Z \propto \int \prod_{\mathbf{k}} d \Re\left(c_{\mathbf{k}}\right) d \Im\left(c_{\mathbf{k}}\right) \Psi_{t}\left\{c_{\mathbf{k}}\right\} \propto \prod_{\mathbf{k}>0} \frac{1}{\mathbf{k}_{\perp}^{2}+\kappa k_{\|}^{2}+\xi^{-2}} .
$$

For the sake of simplicity, the subscript conf of the conformational partition function and of the associated free energy will be dropped from now on.

As a consequence of the occurrence of a confined volume $V$, the density modes with half wavelength larger than $\sim V^{1 / 3}$ are frozen; we will refer to them as "quenched" (or "frozen") modes. The existence of quenched modes can be modeled by simply setting to unity the corresponding factors in the product of Eq. (7). The calculation of $\delta \mu$ is then performed through Eq. (2) by differentiating with respect to the number of appropriate chain units, as shown below.

In light of the representation given in the inset in Fig. 4, the excess chemical potential $\delta \mu$ appearing in Eq. (1) is, in fact, a potential barrier to be overcome in order to allow a chain segment to adopt a new conformation (in fact, the effect of confinement by means of $\delta \mu$ is closely related to the increase of $T_{g}$ usually observed in polymers as crystallinity increases). A segment at the basal interface is able to accommodate into a crystalline cell provided a density fluctuation of suitably large free energy takes place. We shall be dealing, therefore, with a crystal-quasiglass (or else crystalconstrained-amorphous) balance condition between states that a segment may occupy, either in the crystalline phase or in the amorphous phase at the top of the barrier.

\section{ANALYSIS OF STRUCTURAL DATA}

\section{A. Vitrification in slab geometry}

The application of the general scheme outlined in the preceding section requires the knowledge of the structural features of the semicrystalline ordered domains in our samples.

Regarding isotropic PET, the preceding structural data were derived from the interface distribution function analysis of the SAXS patterns. ${ }^{15}$ For cold-drawn PET, instead, the available data were obtained by multiplying the volume crystallinity $\alpha_{V}$ by the long period $L$ derived from the SAXS patterns. ${ }^{4}$ This procedure relies on the assumption that, in oriented PET, volume and linear crystallinity values are close 




FIG. 5. Gibbs-Thomson-like plot for cold-drawn (Table I) and unoriented (Refs. 15 and 17) annealed PET samples. The $l_{c}^{-1}=0$ intercept $T_{m}^{\infty}$, extrapolated by the linear fit to the $T_{a} \geqslant 160{ }^{\circ} \mathrm{C}$ data, is close to the equilibrium melting point of the literature. $T_{a p p}^{\infty}$ would be an apparent melting point extrapolated by the linear fit to the low- $T_{a}$ data for oriented PET (dotted line).

to each other, that is, that only a very small fraction of amorphous phase remains outside of the ordered semicrystalline structures. The arguments exposed in Sec. III support this assumption.

Figure 5 illustrates the plot of $T_{a}$ vs $l_{c}^{-1}$ after crystallization for cold-drawn and unoriented PET samples. The linear fitting restricted to the data points of the cold-drawn sample within the interval $160{ }^{\circ} \mathrm{C} \leqslant T_{a} \leqslant 240{ }^{\circ} \mathrm{C}$ (dashed line) intercepts the $l_{c}^{-1}=0$ axis at the temperature $T_{m}^{\infty} \simeq 285^{\circ} \mathrm{C}$. This value falls within the range indicated in the literature for the equilibrium melting point of PET. ${ }^{16}$ Data collected from isotropic samples are also reported in Fig. 5 and follow roughly the same crystallization line. ${ }^{15,17}$ More recent data on unoriented PET annealed at low $T_{a}$ 's also fit to the dashed crystallization line. ${ }^{18}$

It is noteworthy that in the cold-drawn samples, crystal lamellae grown at $T_{a} \leqslant 140{ }^{\circ} \mathrm{C}$ are thinner than predicted by the low- $T_{a}$ extrapolated linear regression. Moreover, the temperatures $T_{a}$ for which the oriented PET data deviate from the dashed line in Fig. 5 are the same as those that fall within the $\alpha$ dispersion region in Fig. 1. We remember that it is for these $T_{a}$ values that the exothermal component just above $T_{r}$ in DSC is more pronounced (see the arrows in the thermograms in Fig. 3). On the other hand, deviations from the crystallization line at low $T_{a}$ are not observed in isotropic PET and the exothermal contributions above $T_{r}$ in the DSC thermograms are not as pronounced as for cold-drawn, low$T_{a}$ samples (see the inset in Fig. 3 for $T_{a}=120^{\circ} \mathrm{C}$ ).

Since, as is known, lamellar thickening is a relevant mechanism of crystal growth in cold-crystallizing oriented PET (Ref. 19) and structural arrest takes place gradually, the lamellar thickness $l_{c}$ will still result from a balance between bulk and surface contributions associated with stem formation:

$$
l_{c}=\frac{2 v_{c} \sigma_{e}}{\Delta \mu_{a p p}},
$$

where $v_{c}$ is the specific volume in the crystalline phase, $\sigma_{e}$ is the basal surface tension, and

$$
\Delta \mu_{\text {app }} \equiv \mu_{\text {app }}-\mu_{c},
$$

with $\mu_{c}$ being the chemical potential of the appropriate chain units in the crystalline phase. Equation (8) applies independently of the presence $(\delta \mu>0)$ or absence $(\delta \mu=0)$ of constraints.

The influence of a mean chain orientation (i.e., of $\kappa$ ) on $\delta \mu$ leads to the differences found between the lamellar thickness at low $T_{a}$ 's and the corresponding extrapolated $l_{c}$ values in the dashed crystallization plot in Fig. 5. In particular, we can use these structural data to estimate the difference

$$
\left.\overline{\delta \mu} \equiv \delta \mu\right|_{\kappa \simeq 4}-\left.\delta \mu\right|_{\kappa=1}
$$

(remember that $\kappa \simeq 4$ is the orientation factor naturally found for PET after cold drawing) by direct application of Eqs. (1) and (8)-(10):

$$
\overline{\delta \mu}=2 v_{c} \sigma_{e}\left(\frac{1}{l_{c}}-\frac{1}{l_{c 0}}\right),
$$

where $l_{c 0}$ is the lamellar thickness indicated by the low- $T_{a}$ extrapolation of the dashed crystallization line in Fig. 5. The meaning of Eq. (11) is that the deviation of $l_{c}$ from the Gibbs-Thomson prediction $l_{c 0}$ is taken to probe the amount of internal structural constraints rendering the interlamellar regions a glass. It is to be noted that $\left.\delta \mu\right|_{\kappa=4}$ and $\left.\delta \mu\right|_{\kappa=1}$ could be related to the $T_{g}$ increase with the appearance of crystalline domains for the cold-drawn and the initially isotropic samples, respectively. However, in the present case, only the $\left.\delta \mu\right|_{\kappa=4}$ and $\left.\delta \mu\right|_{\kappa=1}$ difference, arising as a consequence of chain orientation, is used.

The crystallization straight line in Fig. 5 associated with the low- $T_{a}$ data of cold-drawn PET (dotted line) intercepts the ordinates at an apparent "equilibrium" crystallization temperature $T_{a p p}^{\infty}<T_{m}^{\infty}$; this temperature would correspond to the crossing of the (imaginary) glass line with the solid line in Fig. 4.

It is convenient to express the factor $2 v_{c} \sigma_{e}$ in Eq. (11) in terms of $l_{c 0}$ by means of Eq. (8) (where, of course, $\mu_{a p p}$ $=\mu_{a}$, since $\delta \mu \equiv 0$ along the crystallization line), using the approximate expression ${ }^{19}$

$$
|\Delta \mu| \simeq H_{f} \frac{T_{m 0}-T}{T_{m 0}},
$$

to estimate the difference $\Delta \mu \equiv \mu_{a}-\mu_{c} ; H_{f}$ in Eq. (12) is the enthalpy of melting and $T_{m 0}$ is the ideal crystal-amorphous equilibrium temperature.

\section{B. Calculation of $\overline{\delta \mu}$ and comparison with the data}

The formation of semicrystalline stacks causes the quenching of all fluctuation modes with wave-vector component, perpendicular to the basal planes, smaller or of the order of 


$$
k_{a} \equiv \frac{\pi}{l_{a}}
$$

where $l_{a}$ is the interlamellar thickness. By setting the corresponding terms in the partition function equal to unity, we can obtain the changes in fluctuation free energy as follows:

$$
\Delta F(\kappa)=F_{q}(\kappa)-F(\kappa) \quad(>0),
$$

where the subscript $q$ indicates the presence of constraints; then,

$$
\overline{\delta \mu}=\left[\frac{\partial}{\partial \mathcal{N}}\left(\left.\delta F\right|_{\kappa=4}-\left.\delta F\right|_{\kappa=1}\right)\right]_{V, T},
$$

with $\mathcal{N}$ is the total number of segments in the volume $V$.

The $k$-space domain $Q$ of the quenched modes depends on the geometry of the interlamellar regions. Assuming that $Q$ is almost the same for both the oriented and the unoriented samples when $T_{a} \leqslant 160{ }^{\circ} \mathrm{C}$ (see below), we find, using Eq. (7),

$$
\left.\delta F\right|_{\kappa=4}-\left.\delta F\right|_{\kappa=1}=k_{B} T \sum_{\mathbf{k}>0}^{\mathbf{k}_{a}} \ln \left[1+\frac{(\kappa-1) k_{\|}^{2}}{\mathbf{k}^{2}+\xi^{-2}}\right],
$$

where $0<\mathbf{k} \leqslant \mathbf{k}_{a}$ is meant to represent the domain $Q$ of the quenched modes. Thus, from Eq. (15) and resorting to the continuum limit $\Sigma_{\mathbf{k}} \rightarrow\left(V / 8 \pi^{3}\right) \int d^{3} \mathbf{k}$, we obtain, after straightforward calculation,

$$
\begin{aligned}
\overline{\delta \mu} \sim & k_{B} T \frac{a v}{\pi^{2}}\left\{k_{a} \ln \left(\frac{\Lambda^{2}+1}{\kappa^{-1} \Lambda^{2}+1}\right)\right. \\
& \left.-\frac{2}{\xi}\left[\frac{1}{\sqrt{\kappa}} \arctan (\Lambda \sqrt{\kappa})-\arctan (\Lambda)\right]\right\},
\end{aligned}
$$

where

$$
\Lambda \equiv k_{a} \xi \text {. }
$$

Note that, for $\kappa>1, \overline{\delta \mu}$ is an increasing function of $\Lambda$, which means that vitrification effects start being significant when the confinement length approaches the correlation length or becomes even smaller.

The temperature dependence of $\overline{\delta \mu}$ is linked to that of $v$ (also through the density autocorrelation length $\xi$ ), which is approximately expressed by ${ }^{7}$

$$
v \approx v_{0}\left(1-\frac{\Theta}{T}\right)
$$

where $\Theta$ is a constant. Thus, $\overline{\delta \mu}$ (and $\xi$ as well) decreases with increasing $T$ and becomes ineffective in the high- $T_{a}$ part of the crystallization line.

For a semiquantitative test of Eq. (17), we assume that the chain orientation is roughly perpendicular to the lamellar basal interfaces. This is not strictly true (see Table I), but at present, we just seek for an agreement with the data on the scale of the order of magnitude.

From the literature data, ${ }^{4,15,18}$ we find that in the annealing temperature interval $100-160{ }^{\circ} \mathrm{C}$, the interlamellar thickness is always $l_{a} \sim 4-5 \mathrm{~nm}$, irrespective of the chain orientation. Now, consider, for instance, the temperature $T_{a}=100{ }^{\circ} \mathrm{C}$. Us- ing Eq. (12), and assuming $H_{f} \simeq 2.5 \mathrm{~kJ} / \mathrm{mol}$ and $T_{m 0} \sim T_{m}^{\infty}$ $=285^{\circ} \mathrm{C},{ }^{16}$ we find $|\Delta \mu| \simeq 0.83 \mathrm{~kJ} / \mathrm{mol}$. On the other hand, using the crystallization line to estimate the lamellar thickness of the unoriented sample, i.e., $l_{c 0} \simeq 2.4 \mathrm{~nm}$, we find an excess chemical potential

$$
|\overline{\delta \mu}|=|\Delta \mu|\left(1-\frac{l_{c}}{l_{c 0}}\right) \simeq 0.6 \mathrm{~kJ} / \mathrm{mol}
$$

(note that $\overline{\delta \mu}$ is not negligible with respect to $\Delta \mu$ ). Since the segment number density is $c \simeq 4.3 \mathrm{~nm}^{-3}$ in the case of PET (assuming that a segment coincides with a monomeric unit), we find that with an orientation parameter $\kappa \simeq 4$ and an interlamellar thickness $l_{a} \sim 4.5 \mathrm{~nm}, \mathrm{Eq}$. (17) predicts the value reported in Eq. (20) provided that the density autocorrelation length is set to a value $\xi \sim 1.3 \mathrm{~nm}$ (i.e., about the length of a monomeric unit ${ }^{4}$ ), that is, the lamellae in oriented PET stop thickening when the condition

$$
\Lambda \simeq 1
$$

is approached.

\section{CONCLUDING REMARKS}

Crystallization studies in polymers often focus on the causes and the conditions controlling growth kinetics and morphology of the crystalline domains or their organization in superstructures. In the present study, the point of view is somewhat reversed, because crystal morphology is used as a probe to investigate the transition of the amorphous domains in the interlamellar regions into a glass during crystallization.

So far, data for PET have been used to test the validity of the model. However, given the generality of the statistical mechanical approach, the picture is open to other semirigid polymer systems. In this respect, it is worth pointing out that further evidence of confinement effects on segmental motion can be drawn by means of the analysis of the relaxation dynamics of the polarization autocorrelation function from dielectric experiments. Reference 9 indeed reports a detailed analysis for PET in terms of a phenomenological model based on the same Adam-Gibbs idea considered above; the scheme, however, is general and its application to other polymeric systems constitutes the subject of our current research.

There are many aspects of the glass transition, in general, which still represent open problems at present, and the experimental observations reported here suggest possible routes to follow so that further progress in this complex field can be made. The following issues represent the main achievements of this work.

(1) We have introduced the concept of vitrification induced by the progressive confinement of the amorphous domains as a consequence of crystal growth. In this scheme, trapping of the defects and quenching of the density fluctuation modes are viewed as two different ways of describing the same mechanism hindering further crystallization. On practical grounds, this idea is expressed through a generalization of a well-known balance equation to the case where the amorphous phase is subjected to dynamic constraints [i.e., Eq. (8)]. An important quantity needed for such a gen- 
eralization is the excess chemical potential $\delta \mu$ associated with the presence of these constraints.

(2) An analytical expression for $\overline{\delta \mu}$ has been derived. Although it contains some approximations, it is attractive because the specific features of the segment structure are contained just in the average bond length $a$ and in the excluded volume interaction parameter $v$. The effects of the structural constraints are expressed in terms of the rather intuitive dimensionless parameter $\Lambda$ [Eqs. (17) and (18)].

(3) The value of the density autocorrelation length $\xi$ $\sim 1.3 \mathrm{~nm}$ is surprisingly close to the characteristic length of cooperativity at the glass transition, derived for unoriented PET by a different method (see Ref. 12). This consistency supports the assumptions adopted throughout the paper.

\section{APPENDIX: OUTLINE OF THE CALCULATIONS}

In the frame of the Gaussian model, the occurrence of a chain conformation $\mathbf{r}(\tau)$, with $\mathbf{r}$ being the position of the segment $\tau$, can be assigned with a probability

$$
\psi=A \exp \left\{-\int_{0}^{n} d \tau\left[\frac{3}{2 a^{2}} \dot{\mathbf{r}}^{2}+\frac{U(\mathbf{r})}{k_{B} T}\right]\right\},
$$

where $\dot{\mathbf{r}} \equiv d \mathbf{r} / d \tau, A$ is a normalization factor, $a$ is the average bond length, $U$ is the energy associated with the position of the segment and $n$ is the number of segments of which the chain is made. Note that $\psi$ as given by Eq. (A1) describes the case where no particular bond orientation is preferred. The integral has the meaning of an energy, consisting of a potential component $U(\mathbf{r})$ and of a contribution (of entropic nature) associated with chain connectivity and proportional to $\dot{\mathbf{r}}^{2}$. Then, a conformational canonical partition function can be assigned to a single chain by a functional integration over all possible paths $\mathbf{r}(\tau)$, i.e., $z_{c o n f} \propto \int \delta \mathbf{r}(\tau) \psi$. This functional integration means that $z_{\text {conf }}$ accounts for all possible fluctuations of the chain shape and, consequently, of the local density.

In order to describe a polymer melt in a volume $V$, a collection of $M$ equal chains with excluded volume interaction, we have to consider the overall chain distribution

$$
\Psi_{t}\left\{\mathbf{r}_{i}(\tau)\right\} \propto \exp \left[-\left(U_{c}+U_{e}\right) / k_{B} T\right],
$$

where

$$
U_{e} \equiv \frac{v}{2} \sum_{i, j=1}^{M} \int_{0}^{n} d \tau \int_{0}^{n} d \zeta \delta\left[\mathbf{r}_{i}(\tau)-\mathbf{r}_{j}(\zeta)\right]
$$

is the excluded volume energy ( $v$ being the interaction parameter) and

$$
U_{c} \equiv k_{B} T \frac{3}{2 a^{2}} \sum_{i=1}^{M} \int_{0}^{n} d \tau\left[\dot{\mathbf{r}}_{\perp i}^{2}+\kappa^{-1} \dot{\mathbf{r}}_{\| i}^{2}\right]
$$

is the connectivity component of the energy allowing for a preferential chain orientation along the direction labeled by $\|$. The orientation parameter $\kappa$ is larger than unity for an oriented melt; the isotropic case is recovered, of course, by setting $\kappa=1$.
From Eqs. (A2)-(A4), the thermodynamics caught by the Gaussian model can be made explicit by functional integration as before. However, it is evident at the outset that in the presence of a crystal-amorphous stack periodicity, not all density (conformation) fluctuations are allowed. In order to account for these constraints easily, it is better to represent $\Psi_{t}$ into the space of density normal modes (that is, in the space of the "collective coordinates," following the nomenclature of Ref. 7), whose amplitudes are defined by

$$
c_{\mathbf{k}}=V^{-1} \sum_{j=1}^{M} \int_{0}^{n} d \tau e^{i \mathbf{k} \cdot \mathbf{r}_{j}(\tau)}
$$

(k being the wave vector), that is, the coefficients $c_{\mathbf{k}}$ 's are the Fourier transforms of the overall segment number density,

$$
c(\mathbf{r})=\sum_{j=1}^{M} \int_{0}^{n} d \tau \delta\left[\mathbf{r}-\mathbf{r}_{j}(\tau)\right] .
$$

The normal-mode representation $\Psi_{t}\left\{c_{\mathbf{k}}\right\}$ of the distribution is the result of the action of a projection operator:

$$
\begin{aligned}
\Psi_{t}\left\{c_{\mathbf{k}}\right\} \propto & \prod_{\mathbf{k}>0} \int \prod_{i} \delta \mathbf{r}_{i} \Psi_{t}\left\{\mathbf{r}_{i}(\tau)\right\} \\
& \times \delta\left[c_{\mathbf{k}}-V^{-1} \sum_{j=1}^{M} \int_{0}^{n} d \tau e^{i \mathbf{k} \cdot \mathbf{r}_{j}(\tau)}\right],
\end{aligned}
$$

where the condition $\mathbf{k}>0$ means that each mode has to be counted once (since $c_{-\mathbf{k}}=c_{\mathbf{k}}^{*}$ ). The delta function reduces $U_{e}$ to a quadratic form in the mode amplitudes:

$$
U_{e}\left\{c_{\mathbf{k}}\right\}=k_{B} T \frac{v V}{2} \sum_{\mathbf{k}} c_{\mathbf{k}} c_{-\mathbf{k}},
$$

whereas for the connectivity contribution $U_{c}$, a representation similar in form can only be taken as an approximation valid for small fluctuations:

$$
U_{c}\left\{c_{\mathbf{k}}\right\} \simeq k_{B} T \sum_{\mathbf{k}} u_{\mathbf{k}} c_{\mathbf{k}} c_{-\mathbf{k}},
$$

with the coefficients $u_{\mathbf{k}}$ related to the average intensity of the corresponding $\mathbf{k}$ mode by

$$
u_{\mathbf{k}}=\frac{1}{2\left\langle c_{\mathbf{k}} c_{-\mathbf{k}}\right\rangle_{0}},
$$

being the average calculated for noninteracting chains (random-phase approximation $^{7,13,20}$ ).

Apart of the anisotropy introduced in the connectivity [cf. Eq. (A4)], the explicit calculation of $\left\langle c_{\mathbf{k}} c_{-\mathbf{k}}\right\rangle_{0}$ follows Ref. 7, with the final result

$$
\left\langle c_{\mathbf{k}} c_{-\mathbf{k}}\right\rangle_{0} \simeq \frac{12 c}{V a^{2}\left(\mathbf{k}_{\perp}^{2}+\kappa k_{\|}^{2}\right)}
$$

valid for large $n$, with $c \equiv M n / V$ being the average segment number density.

With this approximation, the segment distribution takes the form 


$$
\Psi_{t}\left\{c_{\mathbf{k}}\right\} \propto \exp \left[-\frac{V a^{2}}{24 c} \sum_{\mathbf{k}}\left(\mathbf{k}_{\perp}^{2}+\kappa k_{\|}^{2}+\xi^{-2}\right) c_{\mathbf{k}} c_{-\mathbf{k}}\right]
$$

where the parameter $\xi \equiv 1 / \sqrt{8 a v c}$ is the density-density correlation length [see Eq. (5) of the main text].

From Eq. (A12), it is immediate to see that the average intensity of mode $\mathbf{k}$ is

$$
\left\langle c_{\mathbf{k}} c_{-\mathbf{k}}\right\rangle=\frac{12 c}{V a^{2}\left(\mathbf{k}_{\perp}^{2}+\kappa k_{\|}^{2}+\xi^{-2}\right)} .
$$

This can be used to find the profile generated by a $\delta$-like perturbation of the density at the origin from the Fourier integral,

$$
\left\langle\delta c^{2}\right\rangle \equiv\langle c(\mathbf{r}) c(\mathbf{0})\rangle-c^{2}=\frac{V}{8 \pi^{2}} \int d^{3} \mathbf{k}\left\langle c_{\mathbf{k}} c_{-\mathbf{k}}\right\rangle e^{-i \mathbf{k} \cdot \mathbf{r}} .
$$

${ }^{1}$ Handbook of Thermoplastic Polyesters, edited by S. Fakirov (Wiley-VCH, Weinheim, 2002), Vols. 1 and 2.

${ }^{2}$ T. Uchiyama, M. Suyama, M. M. Alam, T. Asano, S. Henning, A. Flores, F. J. Baltá Calleja, and M. F. Mina, Polymer 48, 542 (2007).

${ }^{3}$ A. Bartolotta, G. Di Marco, F. Farsaci, M. Lanza, and M. Pieruccini, Polymer 44, 5771 (2003).

${ }^{4}$ T. Asano, F. J. Baltá Calleja, A. Flores, M. Tanigaki, M. F. Mina, C. Sawatari, H. Itagaki, H. Takahashi, and I. Hatta, Polymer 40, 6475 (1999).

${ }^{5}$ A. Flores, F. J. Baltá Calleja, and T. Asano, J. Appl. Phys. 90, 6006 (2001).

${ }^{6}$ G. Adam and J. H. Gibbs, J. Chem. Phys. 43, 139 (1965).

${ }^{7}$ M. Doi and S. F. Edwards, The Theory of Polymer Dynamics, (Oxford University Press, Oxford, 1998).

${ }^{8}$ T. A. Ezquerra, F. J. Baltá Calleja, and H. G. Zachmann, Polymer 35, 2600 (1994).

${ }^{9}$ M. Pieruccini, T. A. Ezquerra, and M. Lanza, submitted to J. Chem. Phys.

${ }^{10}$ G. Strobl, Prog. Polym. Sci. 31, 398 (2006).
${ }^{11}$ A. A. Minakov, D. A. Mordvintsev, and C. Schick, Polymer 45, 3755 (2004).

${ }^{12}$ C. Schick and E. Donth, Phys. Scr. 43, 423 (1991).

${ }^{13}$ T. A. Vilgis, M. Benmouna, and H. Benoit, Macromolecules 24, 4481 (1991).

${ }^{14}$ P. G. Debenedetti, Metastable Liquids, Concepts and Principles (Princeton University Press, Princeton, NJ, 1996).

${ }^{15}$ C. Santa Cruz, F. J. Baltá Calleja, H. G. Zachmann, N. Stribeck, and T. Asano, J. Polym. Sci., Part B: Polym. Phys. 29, 819 (1991).

${ }^{16}$ Polymer Handbook, 4th ed. edited by J. Brandrup, E. H. Immergut, and E. A. Grulke (Wiley, New York, 1999).

${ }^{17}$ M. Imai, K. Kaji, and T. Kanaya, Macromolecules 27, 7103 (1994).

${ }^{18}$ A. Flores, M. Pieruccini, N. Sribeck, S. S. Funari, E. Bosch, and F. J. Baltá Calleja, Polymer 46, 9404 (2005).

${ }^{19} \mathrm{G}$. Strobl, The Physics of Polymers, 2nd ed. (Springer, New York, 1997).

${ }^{20}$ P. G. De Gennes, Scaling Concepts in Polymer Physics (Cornell University Press, Ithaca, 1996). 